\title{
Genetic defects in protein glycosylation: Introduction
}

\author{
Hans Vliegenthart
}

Published online: 25 December 2012

(C) Springer Science+Business Media New York 2012

The past decades have shown great progress in the unravelling the biosynthetic pathways of protein glycosylation. The identity of the living cell and its compartmentalisation, the structure of the acceptor protein and the availability of sugar-donors, are key parameters in this non-template driven process. The carefully regulated process comprises a number of sequential steps carried out by specific enzymes, affording a glycosylation pattern that is on one hand specific for the protein and on the other for the cell-type. The comparative investigations of species and organs, have disclosed the universality of the main pathways, versus steps characteristic for specific proteins and cells.

In a route wherein so many enzymes at different levels are involved various defects may occur. In the human this feature may give rise to a class of inborn errors of metabolism, known as Congenital Disorders of Glycosylation (CDG), wherein glycosylation is disturbed. The defects may be located at different levels giving rise to various subclasses of disease. The following short reviews present several of the aspects.

As illustrated by Liu and Hirschberg mutations in nucleotide sugar transporters may give rise to global defects in glycosylation. This may lead to diseases as shown for human and bovine cases. Mass spectrometry is today an indispensable tool for the study of structural glycomics and changes therein due to mutations. Wührer presents the application of mass spectrometry to study glycosylation changes in CDG and other diseases. Foulquier et al. focus the attention on glycosylation disorders resulting from defects in membrane trafficking. In particular, alterations in vesicle- formation and -tethering are discussed. In a report by Flanagan-Steet and Steet the functional role of specific classes of glycans during embryonic development in zebrafish are highlighted. The authors stipulate that fundamental work on zebrafish may provide further clues as to glycosylation disorder related diseases. Lauc et al. show the progress that has been made in high throughput glycomics, genomics and epigenomics. This enabled epidemiological and genome-wide association studies of the glycome, providing a wealth of details that are helpful in analysis of the molecular pathology of glycosylation related diseases. Dolichol plays an essential role in protein $\mathrm{N}$-glycosylation. As shown by Welti defects in dolichol pathways may lead to deficiencies in N-glycosylation and thereby to diseases. Katoh and Tiemeyer discuss the progress in insight in $\mathrm{N}$ - and $\mathrm{O}$ glycosylation in Drosophila glycoproteins in relation to vertebrate systems. The results are relevant for a further understanding of pathological features. The progress made in the identification of novel types of CDG by homozygosity mapping and exome sequencing is discussed by Matthijs et al. One of the great challenges in this area is the development of effective therapies. Possible therapeutic approaches toward CDGs are addressed by Thiel and Körner.

This collection of short reviews, written on invitation by Thierry Hennet, review data on the biosynthesis of protein glycosylation and on the pathological aspects of defects in these routes. It is evident that still a lot of fundamental work is necessary to gain further insight in the cause of diseases related to aberrant glycosylation.

Hans Vliegenthart

Editor in Chief

H. Vliegenthart $(\triangle)$

Bijvoet Center, Utrecht University,

Padualaan 8,

3584CH Utrecht, The Netherlands

e-mail: j.f.g.vliegenthart@uu.nl 